\title{
Prescribing antibiotics for urgent dental care during the pandemic
}

The British Dental Association (BDA) has published the following open letter on antibiotic prescribing jointly with Public Health England, the Faculty of General Dental Practice (UK) and the Faculty of Dental Surgery, Royal College of Surgeons of England.

\section{Dear Colleague}

Background

We are in unprecedented times dealing with a global pandemic that is affecting the health and economics of our country and impacting personally on all of $u s$.

We recognise the difficulties for the dental profession in managing patients when there is sustained transmission of COVID-19 and the suspension of routine dental care.

The current Standard Operating Procedure describes the need to reduce the numbers of patients who are seen face to face. The advice for dentists is to remotely triage patients where possible with Advice, Analgesics and Antibiotics where appropriate.

We recognise the challenges which this presents for clinicians in terms of assessment, diagnosis and management, and the effect which this can have on patient care. We appreciate that you are currently operating in the context of treatment via urgent dental care systems, and NHS England have discussed with the regions the need to ensure that acceptance criteria support the prescribing guidance set out in the SOP and this document.

Appropriate prescribing of antibiotics

This (note/brief/letter) seeks to clarify that even in these challenging times, antibiotics still need to be prescribed appropriately for the patients' condition and should follow choice, dose and duration of antibiotic recommended in FGDP(UK) or SDCEP guidance.

- $F G D P(U K)$. Antimicrobial Prescribing for General Dental Practitioners https://www. fgdp.org.uk/guidance-standards

- SDCEP. Drug prescribing for dentistry http://www.sdcep.org.uk/wp-content/ uploads/2016/03/SDCEP-DrugPrescribing-for-Dentistry-3rd-edition.pdf

- SDCEP. Drugs for the Management of Dental Problems During COVID-19

Pandemic http://www.sdcep.org. uk/wp-content/uploads/2020/04/

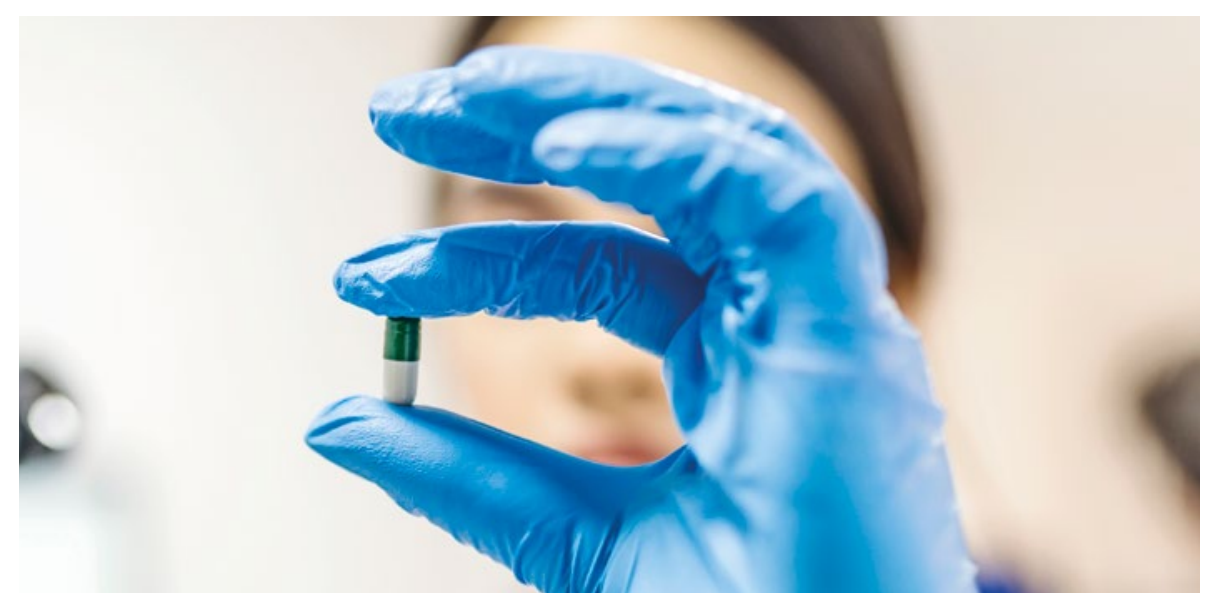

SDCEP-MADP-COVID-19-drugsupplement-080420.pdf

- The dental AMS toolkit also includes patient information leaflets that could be used to provide or reinforce key messages to patients https://www.gov.uk/guidance/ dental-antimicrobial-stewardship-toolkit.

The General Dental Council's 'expectations of dental professionals' falls within Standard 7.1 of its 'Standards for the Dental Team" which requires that 'you must provide good quality care based on current evidence and authoritative guidance'. It also recommends that if there is deviation from established practice and guidance, dentists should record the reasons why and be able to justify their decision.

In relation to the prescribing of medications the GDC offers the following guidance: ${ }^{3}$

- You must make an appropriate assessment of your patient's condition, prescribe within your competence and keep accurate records

- You must have an understanding of your patient's current health and medication, including any relevant medical history, in order to prescribe medicines safely

- You must only prescribe medicines to meet the identified dental needs of your patients.

- You should only use remote means to prescribe medicines for dental patients if there is no other viable option and it is in their best interests.

There is recognition that for many patients, 'remote means' are currently the only option for prescribing drugs.
In summary

Antibiotics should be prescribed in the following situations:

- If it is considered that the patient has a bacterial infection which requires antibiotics. This would include the treatment of acute apical or periodontal abscess and acute pericoronitis, necrotising ulcerative gingivitis/periodontitis

- After discussion with the patient about the benefits and risks associated with the treatment options offered

- With advice on what to do if symptoms continue to progress

- With consideration of a follow-up call to the patient after a few days to check how their infection has responded to the antibiotics.

Antibiotics should not be prescribed:

- To treat conditions that do not respond to antibiotics eg pulpitis

- Because of a patient request

- Routinely for all patients as part of a pathway to care.

\section{References}

1. NHS E/I COVID-19 guidance and standard operating procedure. Urgent dental care systems in the context of coronavirus. Available at: https://www.england.nhs. uk/coronavirus/wp-content/uploads/sites/52/2020/04/ C0282-covid-19-urgent-dental-care-sop.pdf (accessed May 2020).

2. General Dental Council. Standards for the dental team. Available at: https://standards.gdc-uk.org/pages/principle7/principle7.aspx (accessed May 2020).

3. General Dental Council. Guidance on prescribing medicines. Available at: https://www.gdc-uk.org/docs/ default-source/guidance-documents/guidance-on-prescribing-medicines.pdf?sfvrsn=2e82e39c_2 (accessed May 2020). 